\title{
A 4-Dimensional Markov Model for the Evaluation of Radio Access Technology Selection Strategies in Multiservice Scenarios
}

\author{
X. Gelabert, J. Pérez-Romero, O. Sallent, R. Agustí \\ Departament de Teoria del Senyal i Comunicacions \\ Universitat Politècnica de Catalunya (UPC) \\ Barcelona, Spain \\ [xavier.gelabert, jorperez, sallent, ramon]@tsc.upc.edu
}

\begin{abstract}
In order to support the conceptual development of Common Radio Resource Management (CRRM) algorithms, this paper provides an analytical approach to the performance evaluation of Radio Access Technology (RAT) selection procedures in a multi-RAT/multiservice environment. In particular, a 4-Dimensional (4D) Markovian model is devised so as to consider the allocation of voice and data services in a GERAN/UTRAN system. Through the analytical definition of well-established Key Performance Indicators (KPIs) we provide numerical results on the evaluation of a load balancing RAT allocation policy.
\end{abstract}

Keywords- Common Radio Resource Management (CRRM), GERAN, UTRAN, Markov modelling, performance evaluation.

\section{INTRODUCTION}

The increasing number of available Radio Access Technologies (RATs) may allow operators to jointly manage, in the most efficient way, the pool of existing resources provided by each of the individual RATs. This concept is usually referred as Common Radio Resource Management (CRRM) and has attracted a lot of attention in the recent years [1] [2]. In order to properly allocate these common resources, CRRM algorithms are devised. Among others, to select an appropriate RAT for an incoming user requesting a given service is a key CRRM algorithm. Although this problem has been covered in a number of papers, e.g. [3] and [4], the proposed methodology usually relies on system level simulations in order to extract some relevant performance metrics. The analytical approach to the CRRM problem, however, has been less addressed in the literature. To our knowledge, only a few analytical proposals have been developed up to date [5]-[7]. In [5], Lincke et al. propose an analytical approach to the problem of traffic overflowing between several RATs using an M-dimensional Markov model. However, in order to derive a closed form solution by means of applying independence between service types, Markov states in this model indicate the number of sessions of each service that are being carried in whole composite network, but not on which RAT each session is being carried out. In [6], Koo et al. evaluate the separate and common
Erlang capacity of a multi-access/multi-service system. A Markovian approach is also assumed and a closed product form expression is provided [8]. However, this implies that the fractional traffic loads of each service over each system are known. In [7], a near-optimum service allocation is proposed in order to maximize the combined multiservice capacity. The authors assumed a-priory knowledge of the services that need to be allocated, rather than modeling user arrival process.

Our proposed analytical model entails a more flexible framework by assuming that only the total offered traffic to the multi-RAT system for each service is known. Furthermore, our model considers a 4D-state fully describing the allocation of two service types onto two RATs. This approach also allows extending some allocation policies in order to consider state border effects, e.g. enabling policy violations in case of blocking at a given RAT.

The paper is organized as follows. Section II presents the analytical model and the notation that will be used throughout the paper. In section III, a load balancing RAT selection policy is described by means of the proposed model. Section IV presents the performance metrics that will be used to evaluate the behavior of the allocation policy in section V. Finally, section VI deals with the conclusions and future work.

\section{ANALYTIC MODEL}

M-Dimensional Markov models have been widely used in the field of networking to model the behavior of communication networks under varying traffic load conditions. In this paper, we are going to drive our attention on two RATs handled under the $3^{\text {rd }}$ Generation Partnership Project (3GPP): the WCDMA-based UMTS Terrestrial Radio Access Network (UTRAN) and the GSM/EDGE Radio Access Network (GERAN).

In order to account for two service types, voice and data, being served over the aforementioned RATs, GERAN and UTRAN, a 4D Markov chain may be used to model the system's behavior. Let $S_{(i, j, k, l)}$ represent the state in which $i$ voice users and $j$ data users are being served in GERAN; and $k$ voice users and $l$ data users are being served in UTRAN. Bear in mind that

This work is partially funded by the IST-AROMA project and by the COSMOS grant (ref. TEC2004-00518, through the Spanish Ministry of Science and Education and the European Regional Development Fund) 
these indices represent the number of active calls/sessions at a given time.

Since, not all combinations of voice and data users in each of the RATs are possible, let $S$ denote the set of feasible states, depending on $S^{G}$ and $S^{U}$, which are the set of feasible states in GERAN and UTRAN respectively, defined as:

$$
S^{G}=\left\{S_{(i, j, k, l)} \mid 0 \leq f_{(i, j)}^{G} \leq 1, \forall k, l\right\}
$$

and

$$
S^{U}=\left\{S_{(i, j, k, l)} \mid 0 \leq f_{(k, l)}^{U} \leq 1, \forall i, j\right\}
$$

where $f_{(i, j)}^{G}$ and $f_{(k, l)}^{U}$ are the normalized feasibility conditions (hereafter referred simply as feasibility conditions) of GERAN and UTRAN respectively. These feasibility conditions depend on the maximum number of voice and data users that can be simultaneously allocated in each RAT limited by the Call Admission Control (CAC) procedures that provide some minimum QoS requirements. Then, for a given state $S_{(i, j, k, l)}$ to be feasible, it must satisfy $S_{(i, j, k, l)} \in S=S^{G} \cap S^{U}$.

In the following subsections, the state feasibilities for GERAN and UTRAN are presented considering the uplink direction.

\section{A. GERAN State Feasibility}

The resource allocation for voice and data services in GERAN is based on the "capacity on demand" principle. A data user may transmit data over a number of simultaneous packet data channels (PDCH) for both the uplink (UL) and the downlink (DL). Moreover, several data users may be multiplexed over a same PDCH for data transmission. Together with the fact that voice services demand a whole resource (timeslot - TSL), and that they share the same transport media as the data services, resources must be shared in a static or dynamic fashion. Several strategies have been devised for handling these types of traffic [9]. In this paper, and for the sake of simplicity, the Complete Sharing scheme will be adopted. We assume that the total capacity is shared between voice and data traffic with no pre-emption priority.

If $C$ is the total number of available channels (TSLs) for voice and data services in the cell, the maximum number of voice users being served in GERAN, $i$, is upper-bounded by $i \leq C$. Considering the UL direction, we assume that data users are granted with a single channel for each connection (i.e. no multislot capabilities are considered), and that a maximum number of allowed users sharing the same TSL is a system parameter given by $n_{C}$. Then, the maximum number of simultaneous data users being served in GERAN must satisfy $j \leq n_{C} C$. Since voice and data services share the total amount of resources, the previous conditions may be expressed jointly as

$$
0 \leq \frac{i}{C}+\frac{j}{n_{C} C} \leq 1
$$

defining the state feasibility condition for GERAN, $f_{(i, j)}^{G}$.

\section{B. UTRAN State Feasibility}

In UTRAN, the UL load factor ( $\left.\eta_{U L}\right)$ condition must hold in order to ensure that users are granted with the desired capacity for their demanding services. Considering $k$ voice users and $l$ data users being served in UTRAN, the $\eta_{U L}$ condition for a single-cell may be expressed as [10]

$$
0 \leq k \frac{1}{\frac{W / R_{b, v}}{\left(E_{b} / N_{0}\right)_{v}}+1}+l \frac{1}{\frac{W / R_{b, d}}{\left(E_{b} / N_{0}\right)_{d}}+1} \leq \eta_{\max }
$$

With $W$ the chip rate; $R_{b, v}$ and $R_{b, d}$ the bit rate granted to voice and data services; $\left(E_{b} / N_{0}\right)_{v}$ along with $\left(E_{b} / N_{0}\right)_{d}$ the target signal-to-noise ratio after dispreading and decoding for voice and data users; and $\eta_{\max }$ the admission threshold. From (4), and considering $\eta_{\max }=1$, the state feasibility condition of UTRAN, $f_{(k, l)}^{U}$, is straightforward.

\section{CAC and Blocking States}

Once the state space has been defined by means of the feasibility conditions in each RAT, let us define, for the sake of convenience, the set of states in which the acceptance of a new user would force a transition to a state $S_{(i, j, k, l)} \notin S$. Under these circumstances, the RAT in question is said to be in a blocking state. Let $S_{b, \sigma}^{\rho}$ denote the set of feasible states where the acceptance of a service type $\sigma$ user in RAT $\rho$ forces the state to move to a non feasible state. Then, the fractional blocking states for voice and data services, i.e. $\sigma=\{v, d\}$, in UTRAN and GERAN RATs, $\rho=\{G, U\}$, are defined as

$$
\begin{aligned}
S_{b, v}^{G} & =\left\{S_{(i, j, k, l)} \in S \mid S_{(i+1, j, k, l)} \notin S\right\} \\
S_{b, d}^{G} & =\left\{S_{(i, j, k, l)} \in S \mid S_{(i, j+1, k, l)} \notin S\right\} \\
S_{b, v}^{U} & =\left\{S_{(i, j, k, l)} \in S \mid S_{(i, j, k+1, l)} \notin S\right\} \\
S_{b, d}^{U} & =\left\{S_{(i, j, k, l)} \in S \mid S_{(i, j, k, l+1)} \notin S\right\}
\end{aligned}
$$

Let $S_{b, \sigma}$ denote the set of feasible states where the acceptance of a user with service type $\sigma=\{v, d\}$ in any RAT $\rho$ forces the state to move to a non feasible state. Then, the blocking states for voice and data, $\sigma=\{v, d\}$, are respectively

$$
S_{b, v}=S_{b, v}^{G} \cap S_{b, v}^{U} \quad \text { (6) and } \quad S_{b, d}=S_{b, d}^{G} \cap S_{b, d}^{U}
$$

\section{State Transitions and RAT Selection Policies}

Transitions between feasible states happen due to call/session arrivals, with arrival rates $\lambda_{v}$ and $\lambda_{d}$ respectively, or due to call/session departures, with service rates $\mu_{v}$ and $\mu_{d}$ respectively. It is (widely) assumed that arrivals are generated according to a Poisson process, and that service times are exponentially distributed.

Given arrival rates $\lambda_{v}$ and $\lambda_{d}$ the RAT selection policies will 
determine the fractional arrival rates into each of the available RATs. Mathematically speaking, given a RAT selection policy $\pi$ we have

$$
\begin{aligned}
\pi: \mathbb{R}^{2} & \longrightarrow \mathbb{R}^{4} \\
\quad\left(\lambda_{v}, \lambda_{d}\right) \longrightarrow & \longrightarrow\left(\lambda_{v}^{G}, \lambda_{d}^{G}, \lambda_{v}^{U}, \lambda_{d}^{U}\right)
\end{aligned}
$$

In this paper, only transitions between neighboring states (those that only differ in a single increment/decrement in a sole state dimension) are allowed. This prevents more than one call/session to arrive or depart from a given state at the same time.

The proposed analytical approach allows us to define a wide range of RAT selection policies taking into account several allocation criterions, such as service type, load, etc. It also enables the definition of main KPIs in order to provide comparison metrics between different RAT selection policies. For brevity purposes, a load balancing RAT selection policy will be used to illustrate the behavior of our proposed model, however other RAT selection schemes may be found in [11].

\section{LoAd Balancing RAT Allocation and State TRANSITIONS}

The load balancing (LB) policy, denoted as $\pi_{L B}$, intends to allocate users to the RAT that undergoes a lower load situation at a given time. In particular, transitions between a source state and possible destination states will depend on the measured load at each destination state. Before expressing this notion in terms of transition rates in our Markov model, it is convenient to define the load metrics in both RATs.

In GERAN, the TSL utilization factor, [13], may be used to measure the load in a given state $S_{(i, j, k, l)} \in S$ as:

$$
L_{(i, j)}^{G}=\frac{n_{(i, j)}}{C}
$$

where $C$ is the total number of available channels (TSLs) in the cell devoted to voice and data traffic services and $n_{(i, j)}$ is the number of occupied channels (TSLs) when $i$ voice users and $j$ data users are currently being served in GERAN. For the case of data users requiring a single slot for their uplink connection, $n_{(i, j)}=\min (C, i+j)$.

The load in UTRAN is calculated by means of the uplink load factor as:

$$
L_{(k, l)}^{U}=k \frac{1}{\frac{W / R_{b, v}}{\left(E_{b} / N_{0}\right)_{v}}+1}+l \frac{1}{\frac{W / R_{b, d}}{\left(E_{b} / N_{0}\right)_{d}}+1}
$$

In order to determine whether the incoming user demanding a given service should be allocated to GERAN or to UTRAN, we define the following help functions:

$$
\begin{aligned}
& \gamma_{(i, j, k, l)}= \begin{cases}1 & \text { if } L_{(i+1, j, k, l)}^{G} \leq L_{(i, j, k+1, l)}^{U} \\
0 & \text { if } L_{(i+1, j, k, l)}^{G}>L_{(i, j, k+1, l)}^{U}\end{cases} \\
& \beta_{(i, j, k, l)}=\left\{\begin{array}{lll}
1 & \text { if } L_{(i, j+1, k, l)}^{G} \leq L_{(i, j, k, l+1)}^{U} \\
0 & \text { if } L_{(i, j+1, k, l)}^{G}>L_{(i, j, k+1, l+1)}^{U}
\end{array}\right.
\end{aligned}
$$

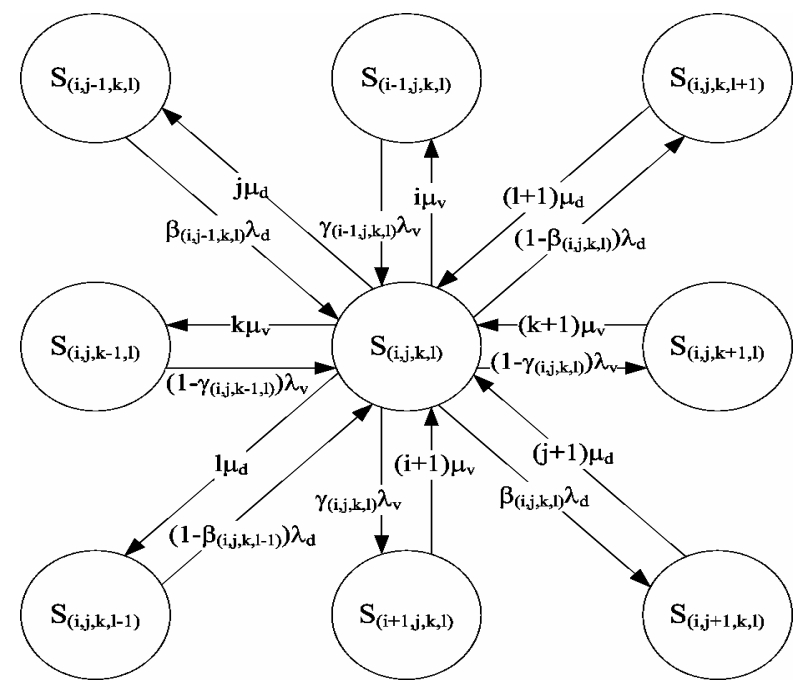

Figure 1 State transition diagram for the general state for policy $\pi_{L B}$.

Then, the associated arrival rates to each RAT may be expressed as a function of $\gamma_{(i, j, k, l)}$ and $\beta_{(i, j, k, l)}$ as:

$$
\begin{aligned}
& \pi_{L B}: \mathbb{R}^{2} \longrightarrow \mathbb{R}^{4} \\
& \quad\left(\lambda_{v}, \lambda_{d}\right) \longrightarrow\left(\gamma_{(i, j, k, l)} \lambda_{v}, \beta_{(i, j, k, l)} \lambda_{d},\left(1-\gamma_{(i, j, k, l)}\right) \lambda_{v},\left(1-\beta_{(i, j, k, l)}\right) \lambda_{d}\right)
\end{aligned}
$$

The state transition diagram at a given generic state $S_{(i, j, k, l)} \in S$ is illustrated in Figure 1.

For convenience, let us define the indicator function $\delta_{(i, j, k, l)}$ which will guarantee that non-feasible states are not taken into account in the balance equations:

$$
\delta_{(i, j, k, l)}= \begin{cases}1 & \text { if } S_{(i, j, k, l)} \\ 0 & \text { otherwise }\end{cases}
$$

By inspection of Figure 1 and regarding the state feasibilities, we may deduce the balance equation for state $S_{(i, j, k, l)} \in S$ as:

$$
\begin{aligned}
& P_{(i, j, k, l)}\left[\gamma_{(i, j, k, l)} \lambda_{v} \delta_{(i+1, j, k, l)}+i \mu_{v} \delta_{(i-1, j, k, l)}\right. \\
& +\left(1-\gamma_{(i, j, k, l)}\right) \lambda_{v} \delta_{(i, j, k+1, l)}+k \mu_{v} \delta_{(i, j, k-1, l)}+\beta_{(i, j, k, l)} \lambda_{d} \delta_{(i, j+1, k, l)} \\
& \left.+j \mu_{d} \delta_{(i, j-1, k, l)}+\left(1-\beta_{(i, j, k, l)}\right) \lambda_{d} \delta_{(i, j, k, l+1)}+l \mu_{d} \delta_{(i, j, k, l-1)}\right] \\
& =\gamma_{(i-1, j, k, l)} \lambda_{v} P_{(i-1, j, k, l)} \delta_{(i-1, j, k, l)}+(i+1) \mu_{v} P_{(i+1, j, k, l)} \delta_{(i+1, j, k, l)} \\
& +\left(1-\gamma_{(i, j, k-1, l)}\right) \lambda_{v} P_{(i, j, k-1, l)} \delta_{(i, j, k-1, l)}+(k+1) \mu_{v} P_{(i, j, k+1, l)} \delta_{(i, j, k+1, l)} \\
& +\beta_{(i, j-1, k, l)} \lambda_{d} P_{(i, j-1, k+1, l)} \delta_{(i, j-1, k, l)}+(j+1) \mu_{d} P_{(i, j+1, k, l)} \delta_{(i, j+1, k, l)} \\
& +\left(1-\beta_{(i, j, k, k-1)}\right) \lambda_{d} P_{(i, j, k, l-1)} \delta_{(i, j, k, l-1)}+(l+1) \mu_{d} P_{(i, j, k, l+1)} \delta_{(i, j, k, k+1)}
\end{aligned}
$$

By applying the constraint

$$
\sum_{\forall(i, j, k, l) \mid S_{(i, j, k, l)} \in S} P_{(i, j, k, l)}=1
$$

we can obtain the steady-state probabilities $P_{(i, j, k, l)}$ in order to evaluate some performance metrics of the system.

It is important to notice that this definition of load balancing implies that a data user will not be forced to share a TSL in GERAN unless no resources are left in UTRAN.

\section{Performance Metrics}

In order to compute the steady state probabilities $P_{(i, j, k, l)}$ we must solve the equation given by (15) for all feasible states 
$S_{(i, j, k, l)} \in S$. This may be carried out using numerical methods; in particular, an iterative power procedure will be utilized for such task [12]. Then, performance metrics may be directly derived from the steady state probabilities, $P_{(i, j, k, l)}$.

\section{A. Blocking Probabilities}

Making use of the blocking state sets defined formerly in section II.C, the generalized form of the blocking probability of a service type $\sigma$ in a given RAT $\rho$ may be expressed as:

$$
P_{b, \sigma}^{\rho}=\sum_{\forall(i, j, k, l) \mid S_{(i, j, k, l)} \in S_{b, \sigma}^{\rho}} P_{(i, j, k, l)}
$$

with $\sigma=\{v, d\}$ and $\rho=\{G, U\}$.

If we are interested in the blocking probability of a particular service type $\sigma$ over all the possible RATs, this can be computed as:

$$
P_{b, \sigma}=\sum_{\forall(i, j, k, l) \mid S_{(i, j, k, l)} \in S_{b, \sigma}} P_{(i, j, k, l)}
$$

\section{B. Carried Traffic}

The average carried traffic (average number of users) may also be computed from the steady state probabilities $P_{(i, j, k, l)}$. The fractional average number of $\sigma$-type users in RAT $\rho$ can be derived numerically from:

$$
N_{\sigma}^{\rho}=\sum_{\forall(i, j, k, l) \mid S_{(i, j, k, l)} \in S} x \cdot P_{(i, j, k, l)}
$$

with

$$
x=\left\{\begin{array}{llll}
i & \text { if } & \sigma=v & \rho=G \\
j & \text { if } & \sigma=d & \rho=G \\
k & \text { if } & \sigma=v & \rho=U \\
l & \text { if } & \sigma=d & \rho=U
\end{array}\right.
$$

\section{Load}

Load metrics are also KPIs which can be obtained from the steady state probabilities. Bearing in mind the load definitions given in (9) and (10), the average TSL utilization factor yields

$$
L^{G}=\sum_{\forall(i, j, k, l) \mid S_{(i, j, k, l)} \in S} L_{(i, j)}^{G} \cdot P_{(i, j, k, l)}
$$

and the average uplink load factor may be computed as

$$
L^{U}=\sum_{\forall(i, j, k, l) \mid S_{(i, j, k, l)} \in S} L_{(k, l)}^{U} \cdot P_{(i, j, k, l)}
$$

\section{Peak Throughput}

Throughput definitions are also inherent of the underlying access scheme and are, consequently, defined individually.

\section{1) GERAN Throughput}

The throughput generated at state $S_{(i, j, k, l)}$ can be expressed as

$$
\Gamma_{(i, j)}^{G}=i \cdot \kappa_{v}+j \cdot \kappa_{d} \cdot R F_{(i, j)}
$$

where $\kappa_{v}$ and $\kappa_{d}$ are the voice and data TSL capacities respectively, and $R F_{(i, j)}$ is the Reduction Factor (RF) [13]. The RF accounts for the multiplexing effect of TSL sharing among data users in GERAN. It takes values between 0 and 1 , meaning a very saturated network in the former, and a low loaded network in the latter. The RF for data users demanding a single slot for their transmission can be computed as

$$
R F_{(i, j)}= \begin{cases}0 & \text { if } j=0 \\ 1 & \text { if } 0<j \leq C-i \\ \frac{C-i}{j} & \text { if } j>C-i\end{cases}
$$

Then, the total average throughput in GERAN becomes:

$$
\Gamma^{G}=\sum_{\forall(i, j, k, l) \mid S_{(i, j, k, l} \in S} \Gamma_{(i, j)}^{G} \cdot P_{(i, j, k, l)}
$$

2) UTRAN Throughput

Throughput delivered in UTRAN at a given state $S_{(i, j, k, l)}$ can be calculated as:

$$
\Gamma_{(k, l)}^{U}=k \cdot R_{b, v}+l \cdot R_{b, d}
$$

where $R_{b, \sigma}$ is the granted bit rate of a $\sigma$ service type user.

Then, the average throughput is

$$
\Gamma^{U}=\sum_{\forall(i, j, k, l) \mid S_{(i, j, k, l)} \in S} \Gamma_{(k, l)}^{U} \cdot P_{(i, j, k, l)}
$$

\section{RESUlts}

The performance of the system is evaluated under different offered voice and data traffic loads, $T_{v}$ and $T_{d}$, where $T_{v}=\lambda_{v} / \mu_{v}$ and $T_{d}=\lambda_{d} / \mu_{d}$. The total number of channels available for GERAN is $C=8$, allowing up to $n_{C}=3$ number of simultaneous users sharing the same TSL. Parameters for UTRAN consider a chip rate of $W=3.84$ Mcps and target signal-to-noise ratios for voice and data services of $6 \mathrm{~dB}$ and $5 \mathrm{~dB}$ respectively. Achievable bit rates for both RATs are assumed to be equal with $R_{b, v}=12.2 \mathrm{kbps}$ and $R_{b, v}=44.8 \mathrm{kbps}$ for voice and data services respectively. Under these assumptions, UTRAN exhibits a higher capacity in terms of maximum number of admissible voice and data users as compared to GERAN. For the Markov model validation, a system level simulator written in $\mathrm{C}++$ was developed considering the aforementioned assumptions and performance metrics.

Figure 2 shows the average load in each RAT for several offered traffic mixings. As expected, the load balancing policy attempts to keep load levels equal in both systems.

Figure 3 shows the number of served users of each service in UTRAN and GERAN. Bearing in mind that, with the current parameter setting, GERAN offers a much lower capacity than UTRAN, many more users (approx. 10 times more for voice services) are needed in UTRAN in order to balance the loads. The number of data users in each RAT is kept constant while offered voice traffic lies between 1.2 and 36 Erlangs. For $T_{v}=84$ Erlangs, data users are forced to share TSLs in GERAN, explaining the increase/decrease of data users in GERAN and UTRAN respectively.

Figure 4 shows the blocking probability that voice users undergo for several offered voice and data traffic loads. 


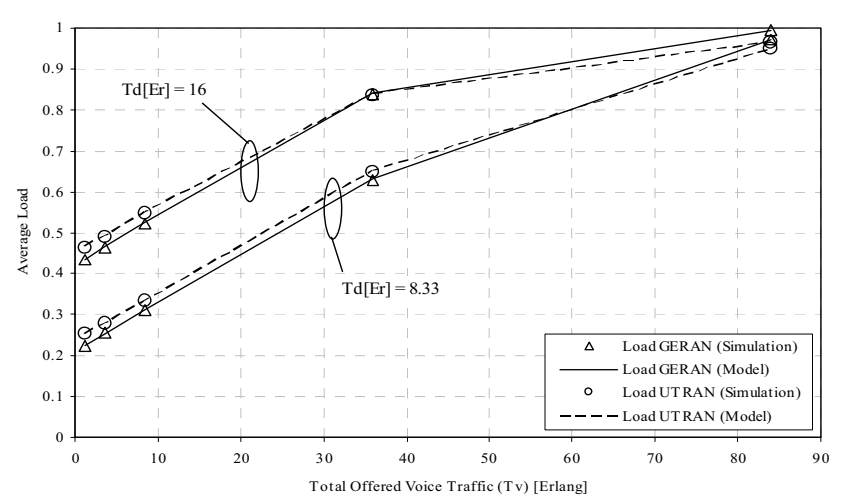

Figure 2 Average Load in each RAT under varying traffic.

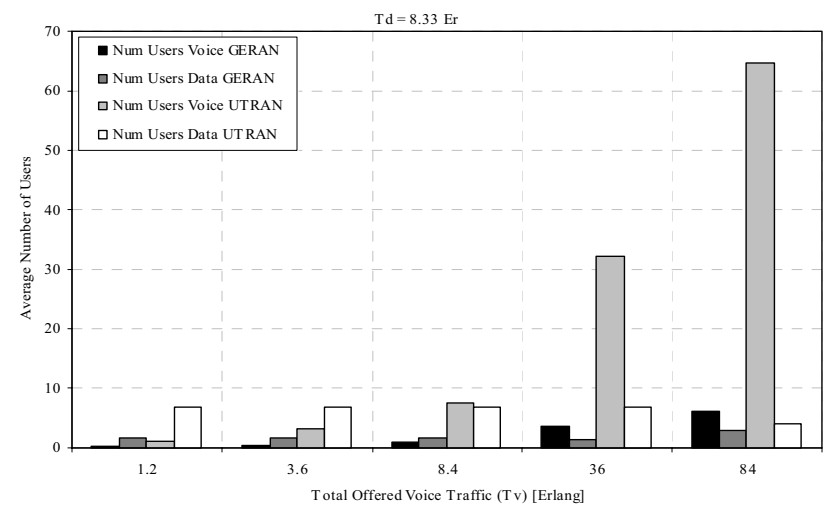

Figure 3 Average number of served users in each RAT.

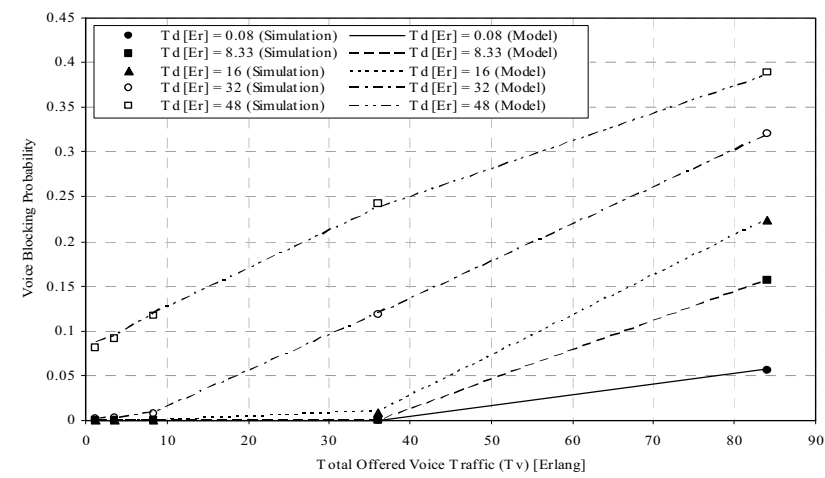

Figure 4 Voice blocking probabilities for each RAT under varying traffic

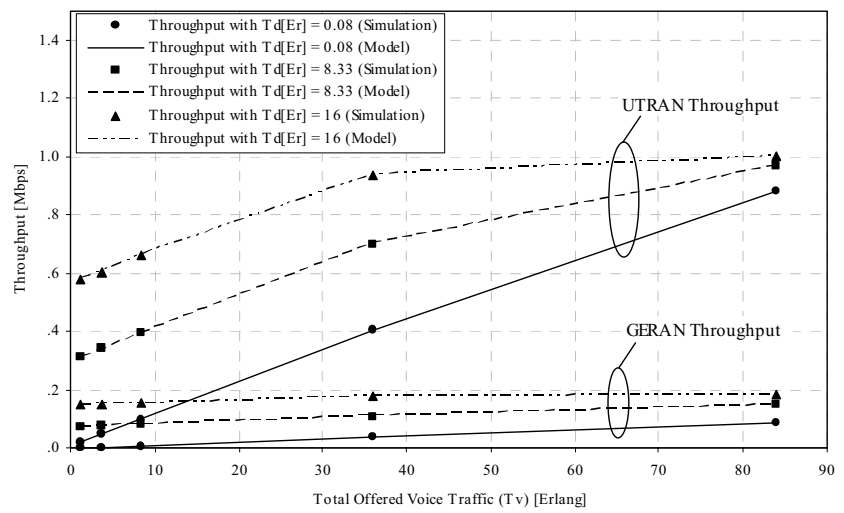

Figure 5 Total throughput per RAT under varying traffic.
Finally, Figure 5 illustrates the total aggregate throughput carried by both RATs when different voice and data loads are offered to the system. Clearly, throughput in UTRAN outperforms the throughput exhibited in GERAN, which is consistent with the different offered capacities in each RAT. Clearly, results obtained via simulation match the ones obtained by means of the analytical model, thus validating the proposed model.

\section{CONCLUDING REMARKS}

In this paper, a 4D Markov chain model has been proposed for the evaluation of RAT selection strategies in a multi-access network comprising GERAN and UTRAN technologies. The model enables a characterization of the main key performance indicators and allows a flexible definition of various RAT selection schemes. In particular, a load balancing scheme has been defined and its behavior evaluated under the considered model. Future work includes the comparison of different RAT selection policies using the same benchmark.

\section{REFERENCES}

[1] 3GPP TR 25.891 v0.3.0, "Improvement of RRM across RNS and RNS/BSS (Post Rel-5) (Release 6)". http://www.3gpp.org/.

[2] J. Pérez-Romero et al. "Common Radio Resource Management: Functional Models and Implementation Requirements", 16th IEEE International Symposium on Personal Indoor and Mobile Radio Communications (PIMRC'05) Berlin, Germany, Sept. 11-15, 2005.

[3] J. Pérez-Romero, O. Sallent, and R. Agustí, "Policy-based Initial RAT Selection algorithms in Heterogeneous Networks" 7th IFIP International Conference on Mobile and Wireless Communication Networks (MWCN'05) Marrakech, Morocco, 2005.

[4] G. Fodor, A. Furuskär, and J. Lundsjo, "On access selection techniques in always best connected networks," in ITC Specialist Seminar on Performance Evaluation of Wireless and Mobile Systems, Aug. 2004.

[5] S. Lincke-Salecker and C. S. Hood, "Integrated Networks That Overflow Speech And Data Between Compnets", International Journal of Network Management, John Wiley \& Sons, July-Aug. 2002, vol 12. issue 4, pp. 235-257.

[6] I. Koo, A. Furuskär, J. Zander and Kiseon Kim, "Erlang capacity of multiaccess systems with service-based access selection," Communications Letters, IEEE, vol.8, no.11pp. 662- 664, Nov. 2004.

[7] A. Furuskar and J. Zander, "Multiservice allocation for multiaccess wireless systems," Wireless Communications, IEEE Transactions on, vol.4, no.1pp. 174- 184, Jan. 2005

[8] R.B. Cooper Introduction to Queuing Theory. Macmillan, NY, 1972.

[9] M. Ermel et al., "Analytical comparison of different GPRS introduction strategies" in Proc. of the 3rd ACM International Workshop on Modeling, Analysis and Simulation of Wireless and Mobile Systems, Boston, MA, US, 2000, pp. 3-10.

[10] J. Pérez-Romero, O. Sallent, R. Agustí, and M. Díaz-Guerra, Radio Resource Management Strategies in UMTS, John Wiley \& Sons, 2005.

[11] X. Gelabert, J. Pérez-Romero, O. Sallent, and R. Agustí, "Performance Evaluation of RAT Selection Strategies in Multiservice/Multi-RAT Scenarios", Internal Report UPC, available on request, (unpublished).

[12] W. J. Stewart, Introduction to the Numerical Solution of Markov Chains, Princeton Univ. Press, Princeton, 1994.

[13] T. Halonen, J. Romero, and J. Melero, GSM, GPRS and EDGE Evolution Towards 3G/UMTS, John Wiley \& Sons, 2000 